\title{
An asymmetric structure of the filament is key to inducing flagellar curvature and enabling motility in the Leptospira spirochete
}

\author{
F. San Martin', K.H. Gibson², F. Trajtenberg ${ }^{1}$, M.R. Brady', E.A. Wunder ${ }^{3}$, M. Picardeau ${ }^{4}$, A. Mechaly ${ }^{1}$, A.I. Ko ${ }^{3}$, \\ C.V. Sindelar ${ }^{2}$, A. Buschiazzo ${ }^{1}$
}

${ }^{1}$ Lab of Molecular \& Structural Microbiology, Institut Pasteur de Montevideo, Mataojo 2020, Montevideo, Uruguay, ${ }^{2}$ Dept. of Molecular Biophysics and Biochemistry, Yale University, 333 Cedar St, New Haven, USA, ${ }^{3}$ Dept. of Epidemiology of Microbial Diseases, Yale School of public Health, 60 College St, New Haven, USA, ${ }^{4}$ Biology of Spirochetes Unit, Institut Pasteur, 28 rue du Dr Roux, Paris, France.

alebus@pasteur.edu.uy

Bacterial flagella are self-assembling nanomachines that enable cell motility by connecting a rotary motor to a long filament. Members of the Spirochaetes phylum, which includes important pathogens, swim using body undulations powered by the rotation of supercoiled flagella that remain confined within the periplasmic space and wrap around the cell body [1]. This behaviour diverges from that of other bacteria, where flagella function as extracellular propellers [2]. Spirochetal filaments are correspondingly distinct in composition and organization, but their molecular structure has remained elusive, obscuring the underlying mechanism for locomotion.

Here we show that, unlike all other known bacterial flagella, a highly asymmetric sheath layer coats the flagellar filament of the Leptospira spirochete and enforces filament supercoiling [3]. We solved 3D structures of wild-type and mutant flagellar filaments from L. biflexa, by integrating customized cryo-electron tomographic averaging methods with X-ray crystallographic analyses of sheath components FcpA and FcpB (Fig. 1). A central core made by FlaB (flagellin-like) protein units, delimits a central $\sim 2 \mathrm{~nm}$ pore.

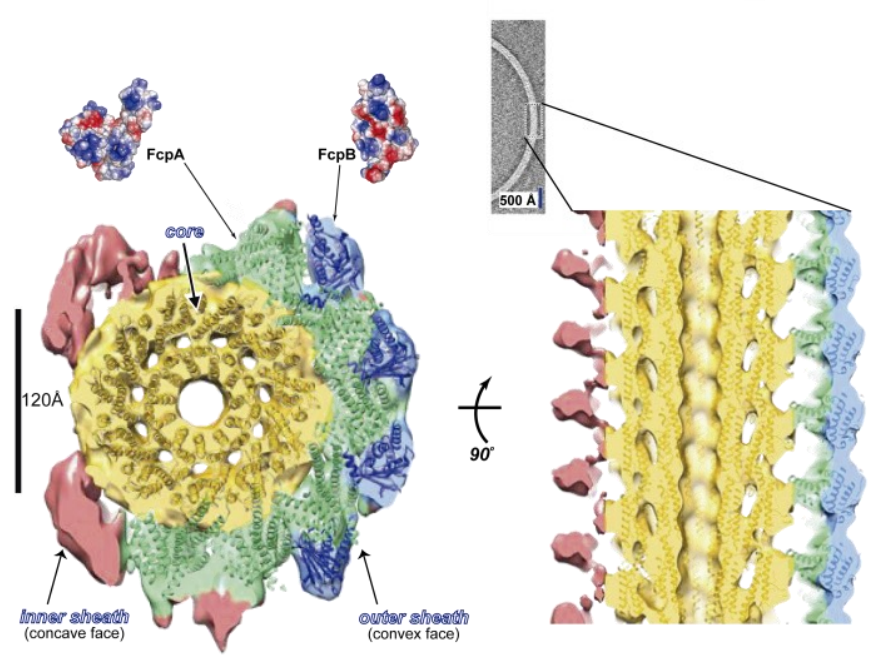

Figure 1. Crystal structures of FcpA and FcpB, fit into the subtomogram-averaged cryo-electron tomography map of $L$. biflexa filaments.

Surrounding the core, FcpA and FcpB proteins co-localize exclusively on the outer, convex side of the filament, as an asymmetric sheath. Distinct sheath components, likely corresponding to FlaA isoforms, instead localize on the concave, inner side of the appendage. Sheath proteins were shown to produce filament supercoiling, an essential feature for flagellar-driven motility [1]. Such radial asymmetry represents a new paradigm of bacterial flagellar architecture, promoting filament supercoiling and ultimately enabling periplasmic flagella to exert their function in Leptospira translational motility. Given the large conservation of most flagellar proteins across the Phylum, this asymmetric filament paradigm might prove valid for other spirochetes.

[1] Charon, N. W., et al. (2012). Annu. Rev. Microbiol. 66, 349.

[2] Erhardt, M., Namba, K. \& Hughes, K. T. Cold Spring Harb. Perspect. Biol. 2, a000299.

[3] Gibson, K. H.*, Trajtenberg, F.*, Wunder, E. A., Brady, M. R., San Martin, F., Mechaly, A., Shang, Z., Liu, J., Picardeau, M., Ko, A. I., Buschiazzo, A. \& Sindelar, C. V. (2020). eLife 9, e53672.

Keywords: bacterial locomotion; endoflagella; integrative structural biology

Acta Cryst. (2021), A77, C291 